\title{
Formulation and Evaluation of Sustained Release Matrix Tablets of Metformin Hydrochloride Using pH Dependent and pH Independent Methacrylate Polymers
}

\author{
Kamlesh J. Wadher ${ }^{1 *}$, Rajendra B. Kakde ${ }^{2}$ and Milind J. Umekar ${ }^{1}$ \\ ${ }^{1}$ Department of Pharmaceutical Technology, Smt. Kishoritai Bhoyar College of Pharmacy, \\ Kamptee, Nagpur - 441002, India \\ ${ }^{2}$ Department of Pharmaceutical Sciences, R.T.M. Nagpur University, \\ Amravati Road, Nagpur - 440033, India.
}

Research Article

Received 24 ${ }^{\text {th }}$ February 2011

Accepted $28^{\text {th }}$ March 2011

Online Ready $8^{\text {th }}$ April 2011

\section{ABSTRACT}

\begin{abstract}
Metformin hydrochloride is recommended globally as first line therapy due to its favorable profile on morbidity and mortality associated with type-2 diabetes mellitus. However, limitations of multiple dosing and risk of triggering gastrointestinal symptoms make its dose optimization difficult. Extended-release metformin matrix tablets were prepared by direct compression of drug and different pH-dependent (Eudragit L-100 and S-100) and $\mathrm{pH}$-independent (Eudragit RLPO and RSPO) polymer combinations. The influence of varying the polymer/polymer (w/w) ratio was evaluated. Among the different examined polymer blends, matrix tablets based on S-100/RLPO and S-100/RSPO mixtures gave the more sustained release pattern. The excipients used in this study did not alter physicochemical properties of the drug, as tested by Fourier transform Infrared Spectroscopy and the thermal analysis using differential scanning calorimetry. All the batches were evaluated for thickness, weight variation, hardness, and drug content uniformity. The in vitro drug dissolution study was carried out using USP 22 apparatus II, paddle method and the release mechanisms were explored. Mean dissolution time is used to characterize drug release rate from a dosage form and indicates the drug release retarding efficiency of polymer. Kinetic modeling of in vitro dissolution profiles revealed the drug release mechanism ranges from diffusion controlled to anomalous type. Fitting the data to Korsmeyer equation indicated that diffusion along with erosion could be the mechanism of drug release.
\end{abstract}


Keywords: Metformin hydrochloride, extended release matrix tablet, $\mathrm{pH}$-independent polymers, $\mathrm{pH}$-dependent polymers, release kinetics;

\section{INTRODUCTION}

Sustained release (SR) drug delivery systems are developed to modulate the release of drug, in order to achieve specific clinical objectives that cannot be attained with conventional dosage forms. Possible therapeutic benefits of a properly designed SR dosage form include low cost, simple processing, improved efficacy, reduced adverse events, flexibility in terms of the range of release profiles attainable, increased convenience and patient compliance (Qiu and Zhang, 2000; Colombo et al., 2000).

Incorporation of drug in the matrix of hydrophilic and hydrophobic polymers have been successfully employed in the development of sustained release delivery systems to provide the desired release profile (Qiu and Zhang, 2000). In recent years, pH-dependent drug delivery systems are considered suitable for designing sustained-drug delivery system (Ashford et al., 1993; Risbud et al., 2000; Palmieri, 2000; Gupta et al., 2000). Amongst the available matrix forming polymers, methacrylic resins (Eudragit $\circledast$ ) appear particularly attractive due to high chemical stability, compactability and variability in physicochemical characteristics (Rodriguez et al., 1993). Several experimental evidences revealed the release retarding ability of ammonio methacrylate (Eudragit $(\mathrm{RL}$ and $\mathrm{RS}$ ) or methacrylic acid copolymers (Eudragit $\AA^{2}$ and $S$ ) in solid dosage forms (Kidokoro et al., 2001; Zhu et al., 2000; Palmieri, 2000; Bruce, 2005).

Due to the variable $\mathrm{pH}$ values observed in the gastrointestinal tract (GIT), the conventional controlled release matrices of ionizable drugs with $\mathrm{pH}$-dependent solubility may give rise to intra- and inter-individual variability in bioavailability (Yamada et al., 1990; Kohri et al., 1992; Vashi et al., 1988). Incorporation of polymers with $\mathrm{pH}$ dependent solubility into controlled release matrix tablets seems to be an obvious approach that provides the desired release over an extended period of time. Hence, appropriate combinations of a $\mathrm{pH}$-dependent polymer with a $\mathrm{pH}$-independent one were suitable for adequately sustaining and controlling the release and assured more reproducible drug release behavior.

Metformin is an oral anti-hyperglycemic agent, shows incomplete absorption from the gastrointestinal tract and the absolute bioavailability is $50-60 \%$ with relatively short plasma half-life of 1.5 - $4.5 \mathrm{~h}$ (Dunn et al., 1995; Defang et al., 2005).

Oral absorption of metformin is confined to the upper part of the intestine, i.e., the duodenum, jejunum and, to a lesser extent, ileum (Vidon et al., 1988) Therefore, the low bioavailability can be ascribed to a comparatively high pre-systemic clearance, due to a lack of correspondence between the time of transit of the drug delivery system, or the free drug, across the upper part of the GI tract and the time required for complete drug absorption. Metformin controlled release may be by preparing formulations able to initiate release in the stomach and complete it in the jejunum, thus realizing a correspondence between time required for complete release and time of transit of delivery system across the upper part of the gastrointestinal $(\mathrm{Gl})$ tract, where drug absorption occurs and where are located sites of metformin glucose-lowering action that contribute to the overall pharmacodynamic effect (Stepensky et al., 2001). 
An obstacle to more successful use of metformin therapy is the high incidence of concomitant gastrointestinal symptoms, such as abdominal discomfort, nausea, and diarrhea that especially occurs during the initial weeks of treatment. Side effects and the need for administration two or three times per day when larger doses are required can decrease patient compliance. A sustained-release (SR) formulation that would maintain plasma levels of the drug for 10 to 16 hours might be sufficient for once-daily dosing of metformin. SR products are needed for metformin to prolong its duration of action and to improve patient compliance (Dunn et al., 1995).

Therefore, the aim of the present work was to evaluate the suitability of different methacryilic resins to prepare direct compression MF matrix tablets able to assure prolonged and well reproducible drug release profiles. In particular, different types of Eudragit $\AA$, i.e. RLPO and RSPO, insoluble but dispersible in water, and L100, S100, with $\mathrm{pH}$-dependent solubility, were used, separately or in different (w/w) combinations, to prepare tablet.

\section{MATERIALS AND METHODS}

\subsection{MATERIALS}

Metformin $\mathrm{HCl}$ was obtained from Universal Medicament Nagpur, India. Microcrystalline cellulose (MCC, Avicel pH 101) was purchased from S. D. Fine Chem. Labs. (Mumbai,India), Eudragit RSPO, Eudragit RLPO, Eudragit S-100 and Eudragit L-100 were obtained as gift samples from Degussa India Ltd. (Mumbai,India). All other ingredients used throughout the study were of analytical grades and were used as received.

\subsection{STUDY OF PHYSICAL INTERACTION BETWEEN DRUG AND POLYMER}

Infrared spectrum was taken by scanning the samples of pure drug and the polymers individually over a wave number range of 4000 to $400 \mathrm{~cm} \mathrm{~cm}^{-1}$ using Fourier transform infrared spectrophotometer (FT-IR, Shimadzu 8400S, Shimadzu, Japan). The change in spectra of the drug in the presence of polymer was investigated which indicates the physical interaction of drug molecule with the polymer.

DSC measurements were performed using a Mettler TA 4000 apparatus equipped with a DSC 25 cell in order to evaluate the drug-excipient compatibility and to verify the absence of solid-state interactions. The thermal analysis was performed in a nitrogen atmosphere at a heating rate of $10^{\circ} \mathrm{C} / \mathrm{min}$ over a temperature range of 30-3008C. Alumina was employed as the reference standard.

\subsection{PREPARATION OF MATRIX TABLETS}

Matrix tablets containing MF. $\mathrm{HCl}$ were prepared by direct compression of binary and ternary drug/polymer(s) mixtures of Eudragit RSPO, RLPO, S-100 and L-100 as shown in table 1. All the ingredients were mixed by a geometric dilution method. Powder were passed through a 20 mesh screen, and then thoroughly mixed with $1 \%$ magnesium stearate. The tablets were compressed using a rotary tableting machine (Rimek Minipress I Ahmadabad, India) with 14-mm flat round punches. A constant compression force was obtained by using the same distance between the upper and lower punches. The binary mixtures were prepared at different drug to- polymer(s) w/w ratios, and ternary systems were all prepared by combining a $\mathrm{pH}$-dependent polymer with a $\mathrm{pH}$-independent one at different drug to polymer(s) ratio 
(1:0.7:0.3, 1:0.5:0.5, and 1:0.3:0.7). Just before compression, the surfaces of the die and punches were lubricated with magnesium stearate. All the tablets were stored in airtight containers for further study.

Table 1. Composition of Various Trial Formulations for the SR metformin $\mathrm{HCl}$ tablet

\begin{tabular}{|c|c|c|c|c|c|c|c|c|}
\hline \multirow[b]{2}{*}{$\begin{array}{l}\text { Formula- } \\
\text { tion code }\end{array}$} & \multicolumn{8}{|c|}{ Ingredients (mg) } \\
\hline & $\begin{array}{l}\text { Metformin } \\
\mathrm{HCl}\end{array}$ & $\begin{array}{l}\text { Eudragit } \\
\text { RSPO }\end{array}$ & $\begin{array}{l}\text { Eudragit } \\
\text { RLPO }\end{array}$ & $\begin{array}{l}\text { Eudragit } \\
\text { S100 }\end{array}$ & $\begin{array}{l}\text { Eudragit } \\
\text { L100 }\end{array}$ & MCC & $\begin{array}{l}\text { Mg. } \\
\text { stea } \\
\text {-rate }\end{array}$ & Total \\
\hline F1 & 500 & 200 & & & & 290 & 10 & 1000 \\
\hline F2 & 500 & 300 & & & & 190 & 10 & 1000 \\
\hline F3 & 500 & & 200 & & & 290 & 10 & 1000 \\
\hline F4 & 500 & & 300 & & & 190 & 10 & 1000 \\
\hline F5 & 500 & & & 200 & & 290 & 10 & 1000 \\
\hline F6 & 500 & & & 300 & & 190 & 10 & 1000 \\
\hline F7 & 500 & & & & 200 & 290 & 10 & 1000 \\
\hline F8 & 500 & & & & 300 & 190 & 10 & 1000 \\
\hline F9 & 500 & 210 & & 90 & & 190 & 10 & 1000 \\
\hline F10 & 500 & 150 & & 150 & & 190 & 10 & 1000 \\
\hline F11 & 500 & 90 & & 210 & & 190 & 10 & 1000 \\
\hline F12 & 500 & 210 & & & 90 & 190 & 10 & 1000 \\
\hline F13 & 500 & 150 & & & 150 & 190 & 10 & 1000 \\
\hline F14 & 500 & 90 & & & 210 & 190 & 10 & 1000 \\
\hline F15 & 500 & & 210 & 90 & & 190 & 10 & 1000 \\
\hline F16 & 500 & & 150 & 150 & & 190 & 10 & 1000 \\
\hline F17 & 500 & & 90 & 210 & & 190 & 10 & 1000 \\
\hline F18 & 500 & & 210 & & 90 & 190 & 10 & 1000 \\
\hline F19 & 500 & & 150 & & 150 & 190 & 10 & 1000 \\
\hline F20 & 500 & & 90 & & 210 & 190 & 10 & 1000 \\
\hline
\end{tabular}

\subsection{EVALUATION OF TABLETS}

The prepared matrix tablets were characterized immediately after preparation for hardness, weight variation, thickness, friability and drug content. The weight variation of the tablets was evaluated $(n=20)$ tablets using an electronic balance (Sartorius GC 103). The hardness of the tablets $(n=6)$ was tested using a Monsanto hardness tester (Campbell Electronics, India). Friability $(n=10)$ was determined in a Roche friabilator (Campbell Electronics, India) for 4 minutes at a speed of $25 \mathrm{rpm}$. (Campbell Electronics, India). The thickness of the tablets 
was measured by vernier caliper. Drug content was analyzed by measuring the absorbance of standard and samples at $\lambda=233 \mathrm{~nm}$ using UV/Visible spectrophotometer (Shimadzu 1601, Kyoto, Japan).

\subsection{IN- VITRO DRUG RELEASE STUDIES}

Drug release studies were conducted using USP-22 dissolution apparatus-2, paddle type (Electrolab, Mumbai, India) at a rotational speed of $50 \mathrm{rpm}$ at $37 \pm 0.5^{\circ} \mathrm{C}$. The dissolution media used were $900 \mathrm{~mL}$ of $0.1 \mathrm{~mol} / \mathrm{L} \mathrm{HCl}$ for first $2 \mathrm{~h}$ followed by $\mathrm{pH} 6.8$ phosphate buffer solutions for $12 \mathrm{~h}$. Sink condition was maintained for the whole experiment. Samples $(10 \mathrm{~mL})$ were withdrawn at regular intervals and the same volume of prewarmed $\left(37 \pm 0.5^{\circ} \mathrm{C}\right)$ fresh dissolution medium was replaced to maintain the volume constant. The samples withdrawn were filtered through a $0.45 \mu$ membrane filter (Nunc, New Delhi, India) and the drug content in each sample was analyzed after suitable dilution with a UV spectrophotometer (Shimadzu UV-1700) at $233 \mathrm{~nm}$ (Gibaldi and Feldman, 1967). The dissolution test was performed in triplicate. Drug dissolved at specified time periods was plotted as cumulative percent release versus time $(h)$ curve.

\subsection{KINETIC ANALYSIS OF RELEASE DATA}

Generally, zero order kinetics, first order kinetics, Hixson-Crowell, Higuchi and KorsmeyerPeppas models were used for the analysis of the dissolution mechanism. When these models are used and analyzed in the preparation, the rate constant obtained from these models is an apparent rate constant. The release of drugs from the matrix tablets can be analyzed by release kinetics theories (Hixson and Crowell, 1931; Higuchi, 1963; Gibaldi and Feldman, 1967 Korsmeyer et al., 1983; Costa and Lobo., 2001). To describe the kinetics of drug release from matrix tablets, release data was analyzed according to Kosmeyer et al's (1983) equation as

Where,

$$
\mathrm{M}_{\mathrm{t}} / \mathrm{M}_{\infty}=K \mathrm{t}^{\mathrm{n}}
$$

$$
\begin{aligned}
& M_{t} / M_{\infty}=\text { fraction solute release } \\
& t \quad=\text { release time } \\
& K=\text { kinetic constant characteristic of the drug/ polymer system } \\
& n \quad=\text { exponent that characterizes the mechanism of release of traces }
\end{aligned}
$$

Based on various mathematical models, the magnitude of the release exponent " $n$ " indicates the release mechanism (i.e. Fickian diffusion, case II transport, or anomalous transport). In the present study, the limits considered were $n=0.5$ (indicates a classical Fickian diffusioncontrolled drug release) and $n=1$ (indicates a case II relaxational release transport; nonFickian, zero-order release). Values of $\mathrm{n}$ between 0.5 and 1 can be regarded as an indicator of both phenomena (drug diffusion in the hydrated matrix and the polymer relaxation) commonly called anomalous transport (Costa et al., 2001).

In order to compare the release profile of different formulas with possible difference in release mechanisms ( $\mathrm{n}$ values), a mean dissolution time (MDT) (Mockel and Lippold, 1993) was calculated using the following equation.

$$
\operatorname{MDT}=(n / n+1) \cdot K^{-1 / n}
$$

Where $\mathrm{n}=$ release exponent and $\mathrm{k}=$ release rate constant 


\subsection{SIMILARITY FACTOR (f2) ANALYSIS}

To evaluate and compare dissolution data, the dissolution profile was statistically analyzed using dissolution similarity factor $f_{2}$. The equation for calculating $f_{2}$ is given below.

$$
f_{2}=50 \log \left\{\left[1+\frac{1}{n} \sum_{t=1}^{t} W t\left(R_{t}-T_{t}\right)^{2}\right]^{-0.5} \times 100\right\}
$$

Where, $\mathrm{n}=$ numbers of dissolution time point

$\mathrm{W}_{\mathrm{t}}=$ Optional weight factor

$\mathrm{R}_{\mathrm{t}}=$ Reference dissolution point at time $\mathrm{t}$

$T_{t}=$ Test dissolution point at time $t$

In vitro release profile of the marketed metformin sustained release (SR) tablets, (Obimet $\mathrm{SR}$, Abbot) was performed under similar conditions as used for in vitro release testing of the test product for the release of metformin. The similarity factor between the 2 formulations was determined using the data obtained from the drug release studies. The $f_{2}$ value between 50 and 100 suggest that the dissolution is similar. The $f_{2}$ values of 100 suggest that the test and reference profile are identical and as the value becomes smaller, the dissimilarity between releases profile increases.

\subsection{SCANNING ELECTRON MICROSCOPY (SEM)}

Electron micrographs metformin hydrochloride matrix tablets before and after dissolution was obtained using a scanning electron microscope (model JSM T200, Joel Ltd., Japan). The specimens were coated under vacuum with gold in an argon atmosphere prior to observation. The scanning electron microscope was operated at an acceleration voltage of $30 \mathrm{kV}$.

\subsection{STATISTICAL ANALYSIS}

The data was subjected to two ways ANOVA followed by Bonferroni post test for analyzing the statistical difference using the software Graph pad prism (San Diego, CA) and in all the cases $p<0.001$ was considered as significant.

\section{RESULTS AND DISCUSSION}

\subsection{STUDY OF PHYSICAL INTERACTION BETWEEN DRUG AND POLYMER}

FTIR studies revealed that metformin hydrochloride showed two typical bands at 3369 and $3296 \mathrm{~cm}^{-1}$ due to $\mathrm{N}-\mathrm{H}$ primary stretching vibration and a band at $3170 \mathrm{~cm}^{-1}$ due to $\mathrm{N}-\mathrm{H}$ secondary stretching, and characteristics bands at 1626 and $1567 \mathrm{~cm}^{-1}$ assigned to $\mathrm{C}=\mathrm{N}$ stretching. No significant shifts of reduction in intensity of the FTIR bands of metformin hydrochloride were observed as shown in figure 1. 


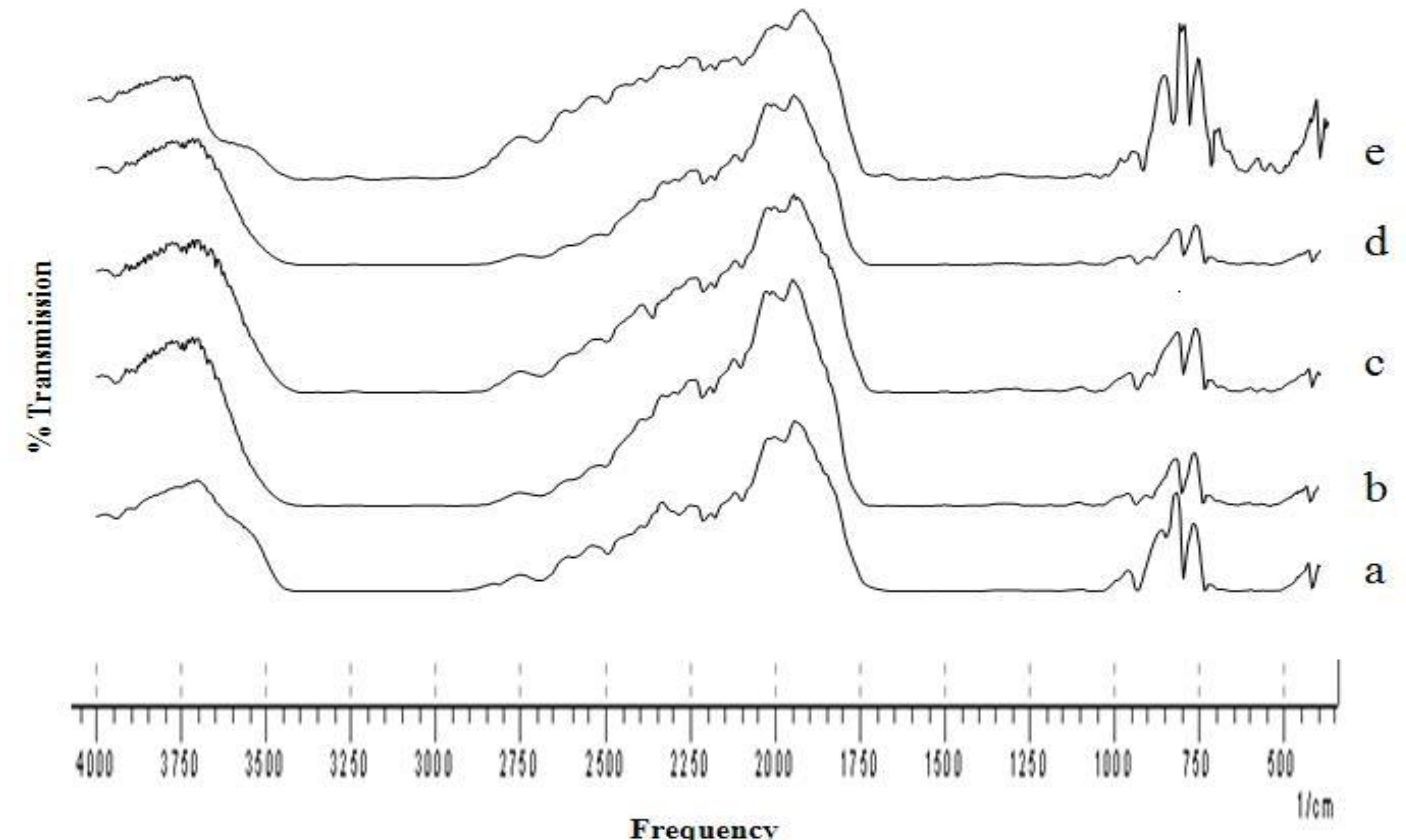

Fig. 1. FT-IR spectra of pure metformin hydrochloride (a), and Physical mixtures of metformin hydrochloride with Eudragit RSPOand S-100 (b), Eudragit RSPO and L-100 (c), Eudragit RLPO and S-100(d), and with Eudragit RLPO and L-100 (e)

DSC analyses were performed in order to evaluate possible solid-state interactions between the components and, consequently, to assess the actual drug-excipient compatibility in all the examined formulations. The thermal curves of pure components and those of some representative ternary systems are shown in Fig 2. The DSC curve of pure Metfrmin exhibited an initially flat profile, followed by a single sharp endothermic peak representing the melting of the substance in the range $223-237^{\circ} \mathrm{C}$ (Tonset $=231.2$, Tpeak $=233.33$ and $\Delta$ Hfusion $=-313.51 \mathrm{~J} / \mathrm{g}$ ). The DSC curves of both Eudragit RLPO and RSPO exhibited a flat thermal profile. The thermal profile of Eudragit L100 and S 100 showed a broad endothermic band ranged between 50 and $100{ }^{\circ} \mathrm{C}$, due to the polymer dehydration, followed by a second endothermic effect at higher temperature, attributable to the melting of its crystalline portion. The DSC curves of both Eudragit RLPO and RSPO exhibited a flat thermal profile, indicative of the completely amorphous nature of these two polymers. The thermal curves of both binary and ternary drug-polymer(s) mixtures, obtained by simple blending corresponded to the superimposition of those of the single components, indicating the absence of solid-state interactions and allowing assessment of drug-polymers compatibility in all the examined formulations. As a further confirmation of the absence of any incompatibility problem, no variations in the thermal behavior of samples of binary and ternary combinations were observed after their tabletting and subsequent powdering. Thus no definite solid-solid interaction could be concluded Examination of all the DSC thermograms. 


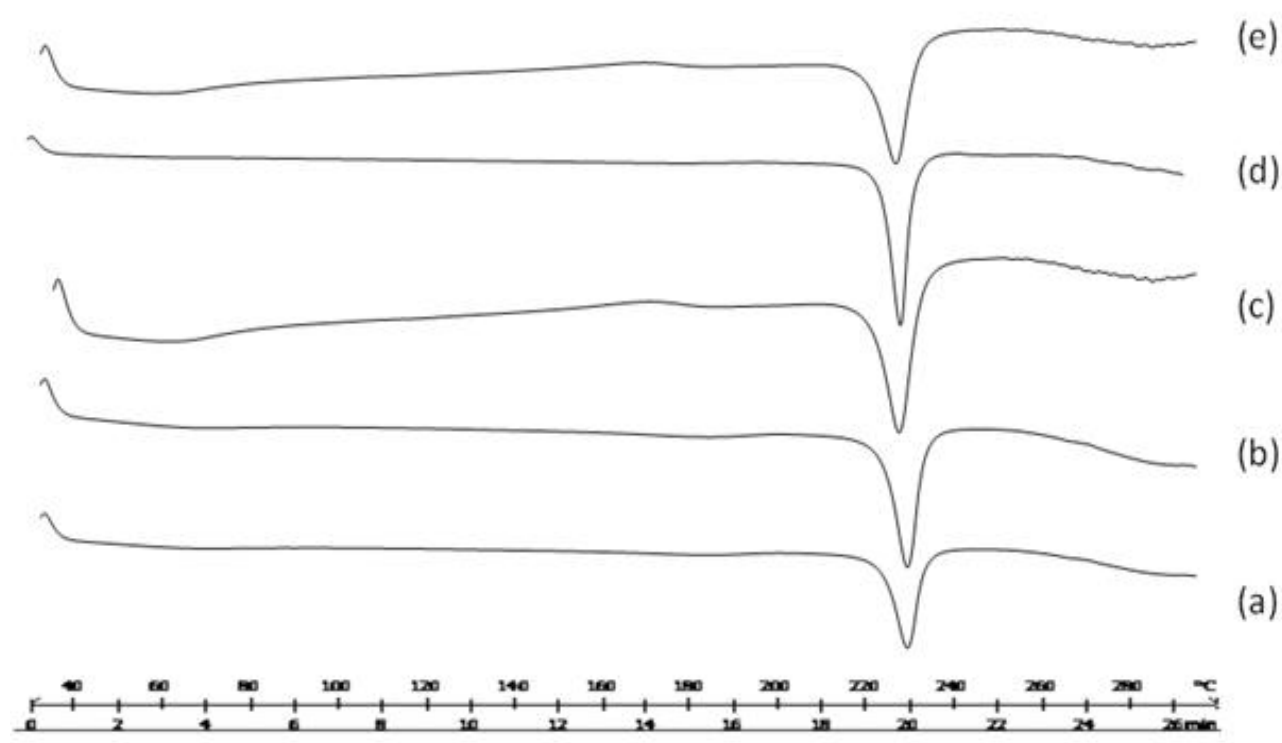

Fig. 2. DSC of pure metformin hydrochloride (a), Ternary mixtures of metformin hydrochloride with Eudragit RSPO with S-100 (b), Eudragit RSPO with L100 (c), Eudragit RLPO with S-100 (d) and Eudragit RLPO and L-100 (e).

\subsection{TABLET CHARACTERISTICS}

The tablet hardness, thickness, weight variations, and friability for each formulation are presented in Table 2. In determinations of tablet weights, all formulations weights were found to be within pharmacopoeia limits. A plain punch with the same radius each time was used for all formulations in tablet pressing, and the differences in tablet radius was not significant $(P<0.05)$.

Friability value of all formulations and commercial tablets were less than $1 \%$, indicates that tablet surfaces are strong enough to withstand mechanical shock or attrition during storage and transportation and until they are consumed (Banker and Anderson, 1987). The average percentage deviation of all tablet formulations was found to be within the above limit, as per official pharmacopoeia requirements (USP, 2000). The manufactured tablets showed low weight variations and a high degree of drug content uniformity among different batches of the tablets, and drug content was more than 95\%. The manufactured tablets showed low weight variations and a high degree of drug content uniformity.

\subsection{DRUG RELEASE STUDIES}

The results of dissolution studies of formulations F1, F2, F3 and F4 composed of binary mixtures of drug and Eudragit RSPO and RLPO (20 and 30\%), respectively are shown in Figure 3, exhibited typical diffusion profiles, characterized by an initial faster release phase followed by a more or less marked decrease in release rate. 
Table 2. Physical properties of the matrix tablets containing $\mathbf{5 0 0} \mathbf{~ m g}$ metformin $\mathrm{HCl}$ as a SR formulation

\begin{tabular}{|c|c|c|c|c|c|}
\hline $\begin{array}{l}\text { Formulation } \\
\text { Code }\end{array}$ & $\begin{array}{l}\text { Hardness } \dagger \\
\text { (kg/cm2) }\end{array}$ & $\begin{array}{l}\text { Friability† } \\
\text { (\%) }\end{array}$ & $\begin{array}{l}\text { Weight } \\
\text { Variation* } \\
\text { (\%) }\end{array}$ & $\begin{array}{l}\text { Drug } \\
\text { Content* } \\
(\%)\end{array}$ & $\begin{array}{l}\text { Thickness } \dagger \\
(\mathrm{mm})\end{array}$ \\
\hline F1 & $7.13 \pm 0.32$ & $0.192 \pm 0.144$ & $1001.28 \pm 5.13$ & 99.13 & $4.44 \pm 0.06$ \\
\hline $\mathrm{F} 2$ & $6.99 \pm 0.58$ & $0.298 \pm 0.251$ & $1001.58 \pm 6.13$ & 99.44 & $4.46 \pm 0.03$ \\
\hline F3 & $8.10 \pm 0.62$ & $0.292 \pm 0.188$ & $1001.18 \pm 5.13$ & 96.23 & $4.39 \pm 0.04$ \\
\hline F4 & $7.88 \pm 0.18$ & $0.258 \pm 0.279$ & $1002.58 \pm 8.13$ & 99.74 & $4.37 \pm 0.05$ \\
\hline F5 & $7.86 \pm 0.38$ & $0.158 \pm 0.279$ & $1001.58 \pm 7.13$ & 99.54 & $4.35 \pm 0.05$ \\
\hline F6 & $7.88 \pm 0.55$ & $0.213 \pm 0.32$ & $1003.26 \pm 6.46$ & 98.34 & $4.54 \pm 0.09$ \\
\hline F7 & $8.01 \pm 0.80$ & $0.211 \pm 0.02$ & $1004.38 \pm 5.13$ & 97.94 & $4.39 \pm 0.08$ \\
\hline F8 & $8.10 \pm 0.48$ & $0.226 \pm 0.12$ & $1002.08 \pm 7.44$ & 96.44 & $4.11 \pm 0.06$ \\
\hline F9 & $7.89 \pm 0.31$ & $0.223 \pm 0.21$ & $1005.28 \pm 6.19$ & 99.34 & $4.18 \pm 0.05$ \\
\hline F10 & $7.88 \pm 0.52$ & $0.158 \pm 0.0$ & $1002.38 \pm 7.35$ & 97.94 & $4.37 \pm 0.06$ \\
\hline F11 & $7.95 \pm 0.59$ & $0.228 \pm 0.11$ & $1000.18 \pm 5.33$ & 96.46 & $4.39 \pm 0.04$ \\
\hline F12 & $7.60 \pm 0.42$ & $0.218 \pm 0.08$ & $1003.53+3.56$ & 98.39 & $4.23 \pm 0.05$ \\
\hline F13 & $7.88 \pm 0.38$ & $0.256 \pm 0.03$ & $1005.38+5.34$ & 99.17 & $4.39 \pm 0.09$ \\
\hline F14 & $7.13 \pm 0.32$ & $0.182 \pm 0.15$ & $1001.68 \pm 5.14$ & 98.13 & $4.24 \pm 0.07$ \\
\hline F15 & $6.98 \pm 0.88$ & $0.248 \pm 0.22$ & $1001.38 \pm 6.44$ & 98.44 & $4.46 \pm 0.03$ \\
\hline F16 & $7.72 \pm 0.15$ & $0.258 \pm 0.13$ & $1005.14 \pm 8.34$ & 97.73 & $4.31 \pm 0.03$ \\
\hline F17 & $8.12 \pm 0.22$ & $0.282 \pm 0.18$ & $1001.58 \pm 7.33$ & 99.23 & $4.30 \pm 0.07$ \\
\hline F18 & $7.88 \pm 0.18$ & $0.248 \pm 0.22$ & $1002.28 \pm 7.13$ & 97.74 & $4.25 \pm 0.06$ \\
\hline F19 & $7.87 \pm 0.25$ & $0.218 \pm 0.33$ & $1002.26 \pm 8.46$ & 99.34 & $4.54 \pm 0.06$ \\
\hline F19 & $8.03 \pm 0.70$ & $0.221 \pm 0.02$ & $1005.18 \pm 3.13$ & 98.34 & $4.38 \pm 0.04$ \\
\hline F20 & $8.05 \pm 0.28$ & $0.236 \pm 0.14$ & $1002.09 \pm 6.13$ & 97.74 & $4.12 \pm 0.04$ \\
\hline
\end{tabular}

$\star A / l$ values are expressed as $M \pm S E, n=20$.

$\dagger$ All values are expressed as $M \pm S E, n=10$

When eudragit RLPO was used as the only retarding polymer for metformin $\mathrm{HCl}$ tablets the entire drug content was released within $2 \mathrm{~h}$ and a sustained drug release pattern was not observed. This might be due to the higher number of quaternary ammonium groups and greater permeability of Eudragit RLPO. When exposed to the dissolution medium, the solvent penetrates into the free spaces between macromolecular chains of Eudragit RLPO. After solvation of the polymer chains, the dimensions of the polymer molecule increase due to polymer relaxation by the stress of the penetrated solvent. This phenomenon may be attributed to surface erosion or initial disaggregation of the matrix tablet prior to gel layer formation around the tablet core. 


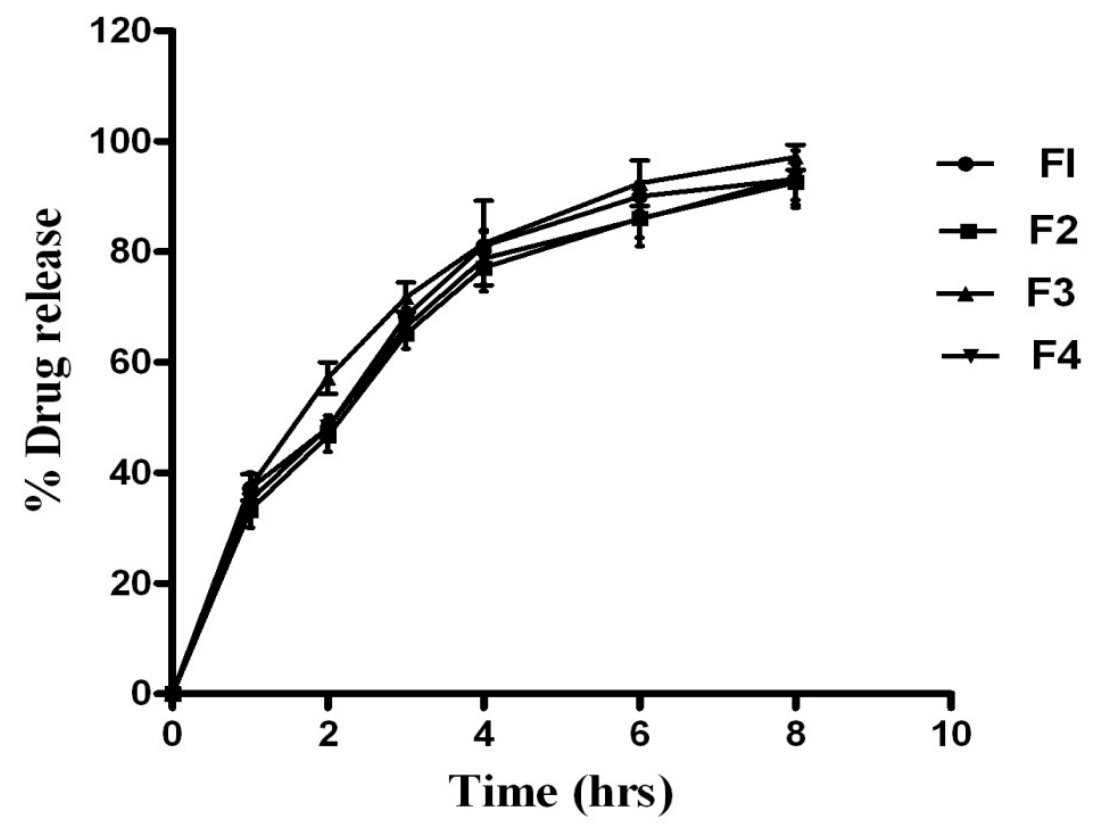

Fig. 3. In vitro cumulative release of Metformin HCL from batches F-1 to F-4 (Each point represents mean $\pm S D, n=3$ )

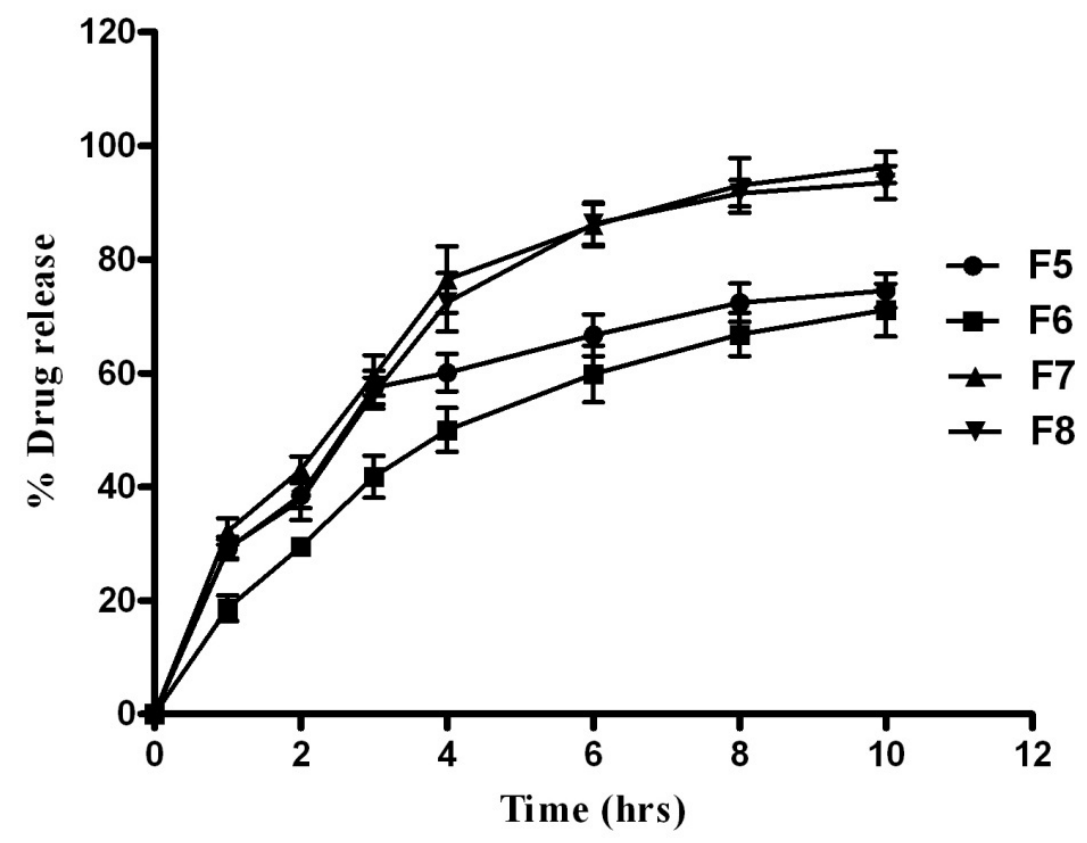

Fig. 4. In vitro cumulative release of Metformin HCL from batches F-5 to F-8 (Each point represents mean $\pm S D, n=3$ ) 
Release profile of formulations F5, F6, F7 and F8 composed of binary mixtures of drug and $\mathrm{pH}$ dependent Eudragit L-100 and S-100 (20 and 30\%) respectively are shown in Figure 4.

Eudragit S100 (F5 and F6) showed the lowest drug release rate (only 74 and $71 \%$ delivered after $10 \mathrm{~h}$ ), being the polymer with the least swelling properties and becoming soluble only at $\mathrm{pH}$ 7. Eudragit L100-matrix tablets (F7 and F8) showed a rather linear release profile (96 and $93 \%$ delivered after $10 \mathrm{~h}$ ). Evidently, in this case the progressive lengthening of the drug diffusion path way was almost counterbalanced by the increase in polymeric matrix solubility at intestinal $\mathrm{pH}$. However, unfortunately, the release behavior from these matrices was not well reproducible, as shown by the error bars, probably due to the irregular erosion process of the matrix.

Matrix tablets were then prepared by using combinations of a $\mathrm{pH}$-dependent Eudragit with a $\mathrm{pH}$ independent one in the different $(0.7: 0.3,0.5: 0.5$ and $0.3: 0.7 \mathrm{w}: / \mathrm{w})$ polymer/polymer ratio (while keeping the total polymer ratio $30 \%$ ), with the aim of obtaining more regular and reproducible release profiles. The release profile of formulations F9, F10, F11 and F12, F13, F14 containing the combination of S-100 and L-100 with Eudragit RSPO (30:70, 50:50 and 70:30 respectively) are shown in figure 5; whereas figure 6 shows the release profile of Formulations F15, F16, F17 and F18, F19, F20 containing the combination of S-100 and L100 with Eudragit RLPO 30:70, 50:50 and 70:30, respectively.

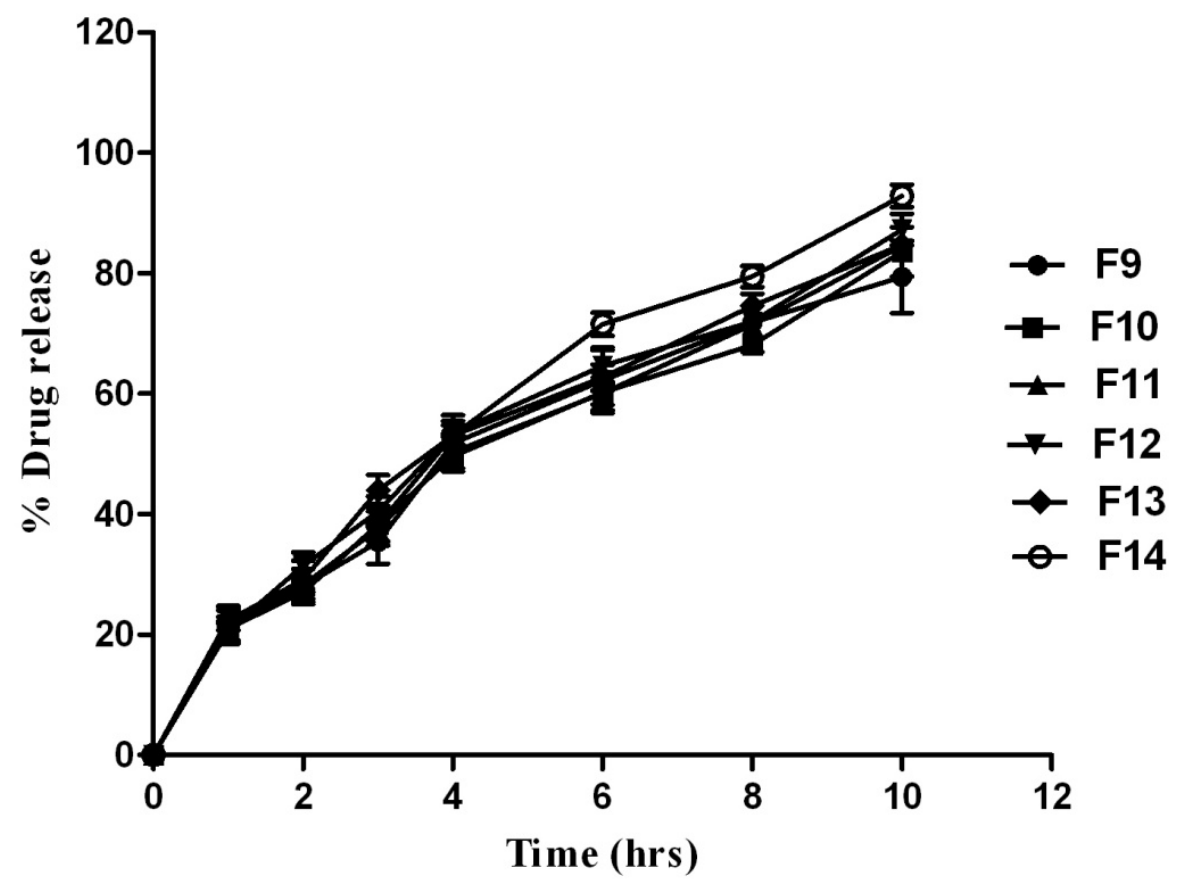

Fig. 5. In vitro cumulative release of Metformin HCL from batches F-9 to F-14 (Each point represents mean $\pm S D, n=3$ ) 


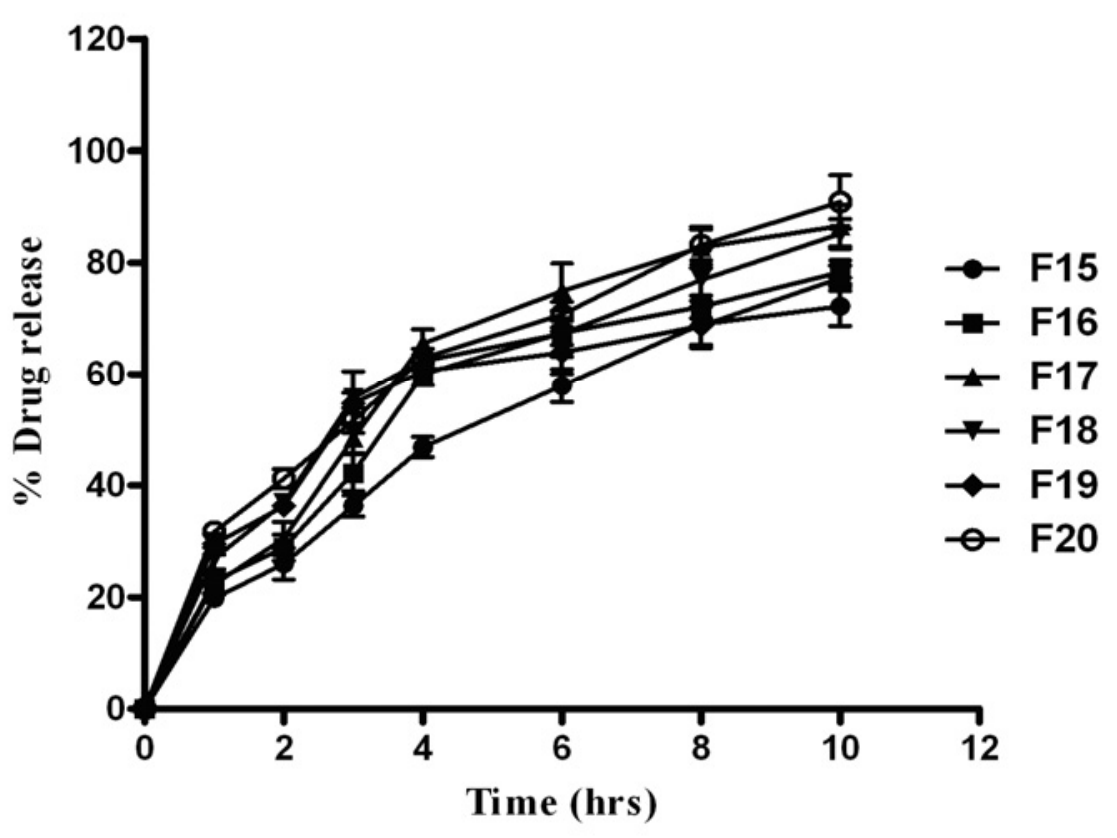

Fig. 6. In vitro cumulative release of Metformin HCL from batches F-15 to F-20 (Each point represents mean $\pm S D, n=3$ )

Among the different examined polymer blends, matrix tablets based on S100/RLPO and S100/RSPO mixtures gave the more sustained release pattern, while the combination of L100/RLPO and L100/RSPO tablets displaying the highest percent of metformin release, but more reproducible results. Tablets with higher content of $\mathrm{pH}$-independent polymer still exhibited a diffusional release profile, being the diffusion process prevailing over the erosion one. On the contrary, tablets with a higher content of the $\mathrm{pH}$-dependent polymer gave the highest percent of final drug released and exhibited more linear release profiles, indicating that the erosion mechanism becomes predominant at intestinal $\mathrm{pH}$. Furthermore, an optimal reproducibility of the drug release behavior was also obtained, according to the error bars (Fig. 5). As for the influence of the kind of $\mathrm{pH}$-independent polymer, RLPO confirmed its better permeability properties than RSPO, as previously observed in tablets prepared using single polymers (Fig. 3). Moreover, the use of such blends of polymers as matrices for tablets allowed the obtainment of more regular release profiles, with the best equilibrium between the values of \% drug released, respectively, at gastric and intestinal $\mathrm{pH}$. This was made possible by the combination of the good erodible properties of $S 100$ and L100 with the swelling ones of RLPO and RSPO polymers.

\subsection{KINETIC ANALYSIS OF RELEASE DATA}

To describe the kinetics of drug release from matrix tablets, release data was analyzed according to different kinetic equations. The data were analyzed by the regression coefficient method and regression coefficient value $\left(r^{2}\right)$ of all batches are shown in Table 3 . 
Table 3. In vitro release kinetics parameters of Metformin HCL from the matrix tablet

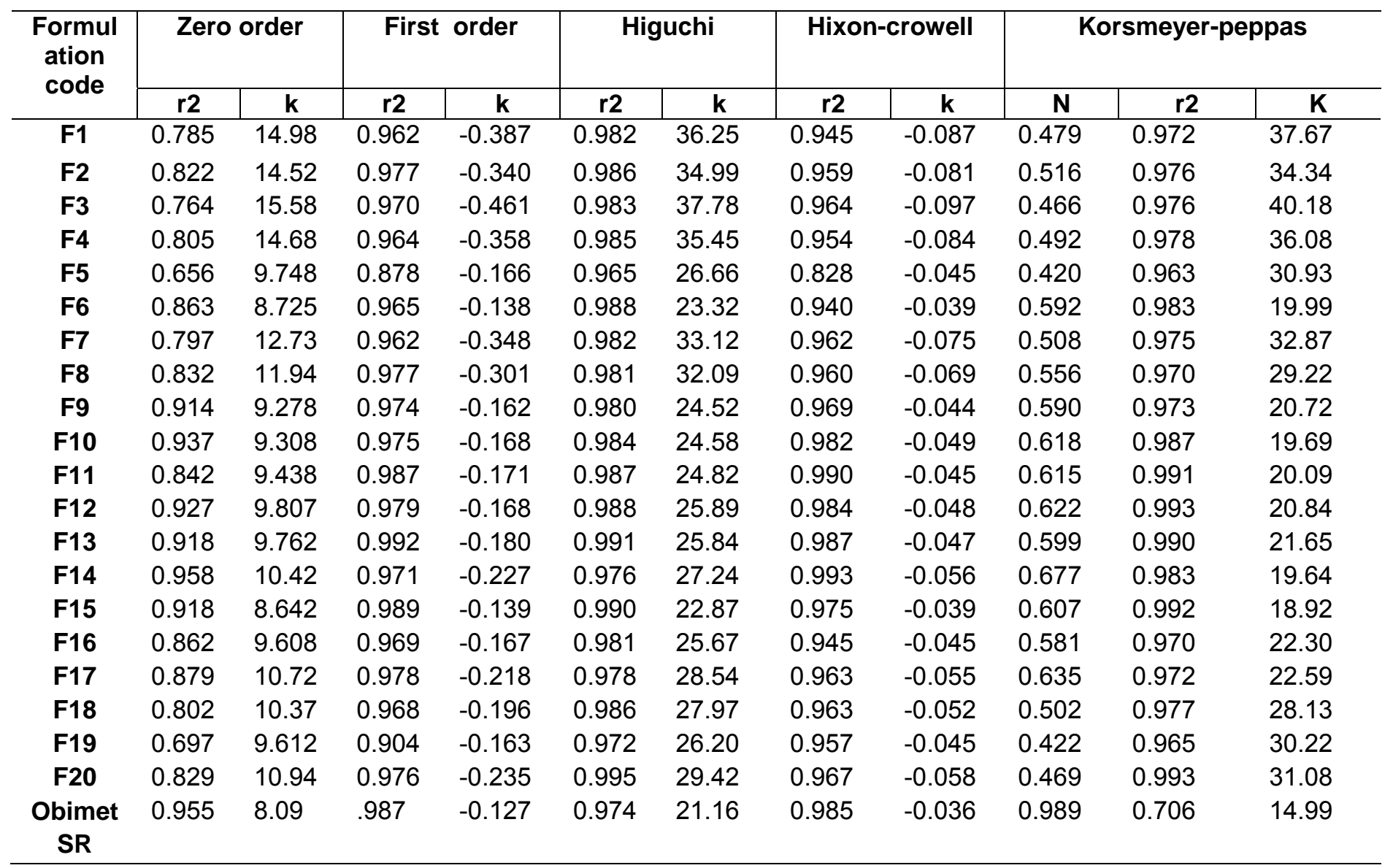


On analyzing regression coefficient values of all batches, it was found that Batch $\mathrm{F} 13$ tablet exhibit almost first order kinetics. Batch F10, F11, F12 and F15 followed Kosermeyr -peppas model; F14 followed Hixon crowell kinetics whereas remaining all the formulations showed higuchi's release kinetics. The in vitro release profiles of drug from all these formulations could be best expressed by Higuchi's equation as the plots showed highest linearity ( $r 2=0.98$ to 0.99) (Korsmeyer et al., 1983). To confirm the diffusion mechanism, the data were fitted into Korsmeyer- Peppas equation (Costa and Lobo, 2001). The formulations showed good linearity ( $\mathrm{r} 2=0.97$ to 0.98 ) with slope $(n)$ between $0.477-0.5879$, which appears to indicate a coupling of diffusion and erosion mechanisms-so called anomalous diffusion.

Table 4. Dissolution Parameter of Sustained Metformin Hcl Matrix Tablets

\begin{tabular}{lllll}
\hline $\begin{array}{l}\text { Formulation } \\
\text { code }\end{array}$ & $\mathbf{t} \mathbf{2 5} \%(\mathbf{h})$ & $\mathbf{t} \mathbf{5 0} \%(\mathbf{h})$ & $\mathbf{t} \mathbf{7 5} \%(\mathbf{h})$ & MDT(h) \\
\hline F1 & 0.5 & 1.9 & 4.3 & 2.11 \\
F2 & 0.5 & 2.0 & 4.6 & 2.32 \\
F3 & 0.4 & 1.8 & 3.9 & 2.12 \\
F4 & 0.5 & 2.0 & 4.5 & 2.29 \\
F5 & 0.9 & 3.5 & 7.9 & 2.38 \\
F6 & 1.1 & 4.6 & 10.3 & 3.12 \\
F7 & 0.6 & 2.3 & 5.1 & 2.68 \\
F8 & 0.6 & 2.4 & 5.5 & 2.72 \\
F9 & 1.0 & 4.2 & 9.4 & 3.56 \\
F10 & 1.5 & 4.5 & 8.7 & 4.06 \\
F11 & 1.4 & 4.4 & 8.5 & 3.96 \\
F12 & 1.3 & 4.1 & 7.8 & 3.84 \\
F13 & 1.6 & 3.8 & 7.7 & 3.69 \\
F14 & 1.6 & 3.7 & 6.6 & 3.95 \\
F15 & 1.6 & 5.0 & 9.7 & 3.38 \\
F16 & 0.9 & 3.8 & 8.5 & 3.09 \\
F17 & 0.8 & 3.1 & 6.9 & 3.06 \\
F18 & 0.8 & 3.2 & 7.2 & 3.12 \\
F19 & 0.9 & 3.6 & 8.2 & 2.84 \\
F20 & 0.7 & 2.9 & 6.5 & 3.21 \\
Obimet SR & 1.4 & 5.6 & 12.6 & 4.10 \\
\hline & & & & \\
\hline
\end{tabular}


The MDT was significantly higher when the combination of $\mathrm{pH}$ dependent polymers with the $\mathrm{pH}$ dependent polymers was carried out than the plain polymers, which clearly indicated sustained release nature of the combination of both Eudragits.

The time taken to release $25 \%\left(t_{25}\right), 50 \%\left(t_{50}\right)$, and $75 \%\left(t_{75}\right)$ of drug from different formulations was determined (Table 4).

Mean dissolution time (MDT) value is used to characterize drug release rate from a dosage form and indicates the drug release retarding efficiency of polymer. The MDT was significantly higher when the combination of $\mathrm{pH}$ dependent polymers with the $\mathrm{pH}$ dependent polymers was carried out than the plain polymers.

\subsection{SIMILARITY FACTOR (f2) ANALYSIS}

Similarity factor analysis between the prepared tablets and Obimet SR tablet for the release of metformin showed an $f 2$ factor $(f 2=64.12)$ greater than 50 which confirms that the release of drug from the prepared tablets was similar to that of the marketed tablet.

\subsection{SCANNING ELECTRON MICROSCOPY (SEM)}

The SEM images of the tablet were taken before and after dissolution as shown in figure 5 .

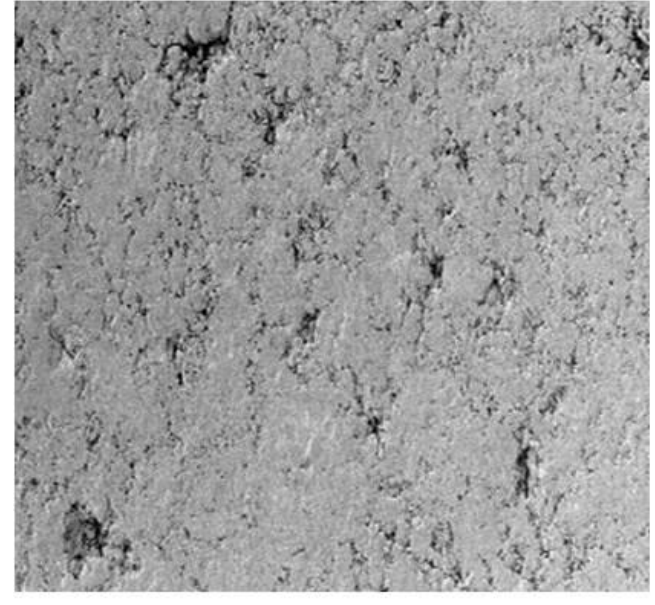

Before dissolution

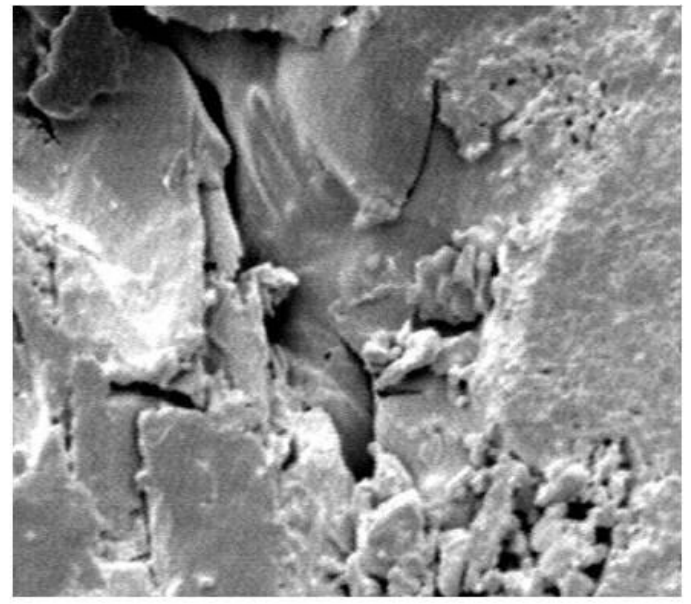

After dissolution

Fig. 7. Scanning electron microscopy image of tablet before and after dissolution

The SEM images of the tablet before dissolution showed intact surface without any perforations, channels, or troughs. After dissolution, revealed many pores with increasing diameter. The solvent front enters the matrix and moves slowly toward the center of the tablet. The drug diffuses out of the matrix after it comes in contact with dissolution medium, which clearly indicates the involvement of both erosion and diffusion mechanisms to be responsible for sustaining the release of metformin from formulated matrix tablets. 


\section{CONCLUSION}

The drug release behavior was markedly influenced by the kind of Eudragit used, and, when utilized in mixtures, by their relative w/w ratio. When eudragit RSPO and RLPO were used as the only retarding polymer for metformin tablet a sustained drug release pattern was not observed. It was found that, appropriate combinations of a $\mathrm{pH}$-dependent polymer with a $\mathrm{pH}$ independent one were suitable for adequately sustaining and controlling metformin release and assured more reproducible drug release behavior. Among the different examined polymer blends, matrix tablets based on S100/RLPO and S100/RSPO mixtures gave the more sustained release pattern. In particular, the use of a mixture of Eudragit $\mathrm{S} 100(\mathrm{pH}-$ dependent) and Eudragit RLPO ( $\mathrm{pH}$-independent) in the 0.3:0.7 w/w ratios enabled a highly reproducible drug release profile to be achieved. The formulations showed good linearity which appears to indicate a coupling of diffusion and erosion mechanisms-so called anomalous diffusion. In view of the importance of metformin hydrochloride in the treatment of diabetes mellitus, the preparation of a suitable sustained release dosage form would not only increase the efficacy of treatment and patient compliance but should also produce desirable blood concentrations and decrease the incidence of adverse effects.

\section{ACKNOWLEDGMENTS}

The authors are thankful to Universal Medicament, Nagpur, India for providing Metformin $\mathrm{HCl}$ as gift sample and S.K.B. College of Pharmacy, Kamptee, Nagpur, India for providing necessary facilities to carry out this work.

\section{REFERENCES}

Ashford, M., Fell, J.T., Attwood, D., Woodhead, P.J. (1993). An in vitro investigation into the suitability of $\mathrm{pH}$-dependent polymers for colon targeting. Int. J. Pharm., 91, 241-245.

Banker, G.S., Anderson, L.R. 1987. Tablets, In: Lachman, L., Liberman, H.A., Kanig, J.L. (Eds.), The Theory and Practice of Industrial Pharmacy. Varghese Publishing House Inc., Mumbai, pp. 293-345.

Bruce, L.D., Shah, N.H., Malick, A.W., Infeld, M.H., McGinity, J.W. (2005). Properties of hotmelt extruded tablet formulations for the colonic delivery of 5-aminosalicylic acid. Eur. J. Pharm. Biopharm., 59, 85-97.

Colombo, P., Bettini, R., Santi, P., Peppas, N.A. (2000). Swellable matrixes for controlled drug delivery: gel-layer behavior, mechanisms and optimal performance. Pharm. Sci. Technol. Today, 3(6), 198-204.

Costa, P., Sousa Lobo, J.M. (2001). Modeling and comparison of dissolution profiles. Eur. J. Pharm. Sci., 13, 123-133.

Defang, O., Shufang, N., Wei, L. (2005). In vitro and in vivo evaluation of two extended Release preparations of combination metformin and glipizide. Drug Dev. Ind. Pharm., 31, 677-685.

Dunn, C.J., Peters, D.H. (1995). Metformin: A review of its pharmacological properties and therapeutic use in non-insulin-dependent diabetes mellitus. Drugs, 49(5), 721-749.

Gibaldi, M., Feldman, S. (1967). Establishment of sink conditions in dissolution rate determinations-theoretical considerations and application to non disintegrating dosage forms. J. Pharm. Sci., 56, 1238-1242.

Gupta, V.K., Beckert, T.E., Price, J.C. (2000). A novel pH- and time based multi-unit potential colonic drug delivery system Development. Int. J. Pharm., 213, 83-91. 
Higuchi, T. (1963). Mechanism of sustained-action medication. Theoretical analysis of rate of release of solid drugs dispersed in solid matrices. J. Pharm. Sci., 52, 1145-1149.

Hixson, A.W., Crowell, J.H. (1931).Dependence of reaction velocity upon surface and agitation. Ind. Eng. Chem., 23, 923-931.

Kidokoro, M., Shah, N.H., Malick, A.W., Infeld, M.H., McGinity, J.W. (2001). Properties of tablets containing granulations of ibuprofen and an acrylic copolymer prepared by thermal processes. Pharm. Develop. Technol., 6, 263-275.

Kohri, N., Miyata, N., Takahashi, M., Endo, H., Iseki, K., Miyazaki, K., Takechi, S., Nomura, A. (1992). Evaluation of $\mathrm{pH}$-independent sustained-release granules of dipyridamole by using gastric-acidity-controlled rabbits and human subjects. Int. J. Pharm., 81, 4958.

Korsmeyer, R.W., Gurny, R., Doelker, E.M., Buri, P., Peppas, N.A. (1983). Mechanism of solute release from porus hydrophilic polymers. Int. J. Pharm., 15, 25-35.

Mockel, J.E., Lippold, B.C. (1993). Zero-order release from hydrocolloid matrices. Pharm. Res., 10, 1066-1070.

Palmieri, G.F., Michelini, S., Martino, P., Martelli, S. (2000). Polymers with pH-dependent solubility: possibility of use in the formulation of gastroresistant and controlled-release matrix tablets. Drug Dev. Ind. Pharm., 26, 837-845.

Quu, Y., Zhang, G. (2000). Research and Development Aspects of Oral Controlled Release Systems, in: Wise, D.L., Klibanov, A.M., Langer, R., Mikos, A.G., Peppas, N.A., Trantolo, D.J., Wnek, G.E., Yaszeski, M.J. (Eds.), Handbook of Pharmaceutical Controlled Release Technology. Marcel Dekker Inc., New York, pp. $465-503$.

Risbud, M.V., Hardikar, A.A., Bhat, S.V., Bhonde, R.R. (2000). pH sensitive freeze-dried chitosan-polyvinyl pyrrolidone hydrogels as controlled release system for antibiotic delivery. J. Control Release., 68, 23-30.

Rodriguez, L., Caputo, O., Cini, M., Cavallari, C., Grecchi, R. (1993). In vitro release of theophylline from directly-compressed matrices containing methacrylic acid copolymers and/or dicalcium phosphate dehydrate. Farmaco., 48, 1597-1604.

Stepensky, D., Friedman, M., Srour, W., Raz, I., Hoffman, A. (2001). Preclinical evaluation of pharmacokinetic-pharmacodynamic rationale for oral CR metformin formulation. J. Control Release, 71, 107-115.

United States Pharmacopeia. (2000). United States pharmacopeia-national formulary (USP 24/NF 19) Rockville: United States Pharmacopeial Convention.

Vashi, V.I., Meyer, M.C. (1988). Effect of pH on the in vitro dissolution and in vivo absorption of controlled-release theophylline in dogs. J. Pharm. Sci. 77, 760-764.

Vidon, N., Chaussade, S., Noel, M., Franchisseur, C., Huchet, B., Bernier, J.J. (1988). Metformin in the digestive tract. Diabetes Res. Clin. Pract., 4, 223-229.

Yamada, I., Goda, T., Kawata, M., Shiotuki, T., Ogawa, K. (1990). Gastric acidity-dependent bioavailability of commercial sustained release preparations of indomethacin evaluated by gastric acidity-controlled beagle dogs. Chem. Pharm. Bull. 38, 31123115.

Zhu, Y., Shah, N.H., Malick, A.W., Infeld, M.H., McGinity, J.W. (2000). Influence of thermal processing on the properties of chlorpheniramine maleate tablets containing an acrylic polymer. Pharm. Develop. Technol., 7, 481-489.

(c) 2011 Wadher et al.; This is an Open Access article distributed under the terms of the Creative Commons Attribution License (http://creativecommons.org/licenses/by/2.0), which permits unrestricted use, distribution, and reproduction in any medium, provided the original work is properly cited. 